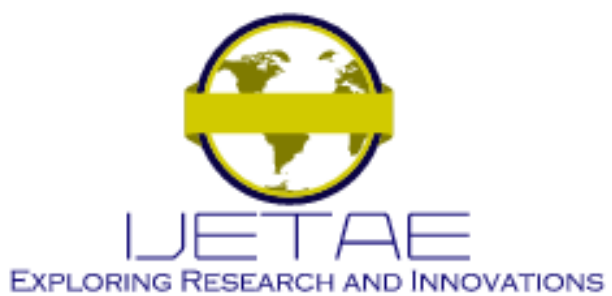

International Journal of Emerging Technology and Advanced Engineering

Website: www.ijetae.com (E-ISSN 2250-2459, Scopus Indexed, ISO 9001:2008 Certified Journal, Volume 12, Issue 02, February 2022)

Manuscript Received: 08 January 2021, Received in Revised form: 07 February 2022, Accepted: 12 February 2022

DOI: 10.46338/ijetae022_20

\title{
Identity Card Detection System using YOLOv3 and Image Rectification
}

\author{
Gede Putra Kusuma ${ }^{1}$, Angelica Faustine ${ }^{2}$, Nikolas Nasir ${ }^{3}$ \\ Computer Science Department, BINUS Graduate Program - Master of Computer Science, Bina Nusantara University, Jakarta, \\ Indonesia, 11480
}

\begin{abstract}
In the age of modern technology like today, object detection is something that is really needed. Object detection is done by considering the type of data collected. This research focused on Identity card detection from an input image taken by a smartphone camera. The Identity card detection is a preliminary step toward automatic identity data collection. Identity data collection is usually performed in a conventional way. This way leads to several problems such as bad data result, unreliable data verification, and long duration on confirming data validity. In this project, we will make an object detection system with YOLOv3 algorithm, and then the result will be used for an edge detection with Canny algorithm and image rectification. The testing result show that YOLOv3 algorithm could reach $92.59 \mathrm{mAP}$ with $5 \mathrm{~s}$ detection time. While the corner detection for image rectification manage to get an average error of $\mathbf{3 7 . 0 6}$ pixels. The establishment of this model has important practical significance for improving Identity Card detection process.
\end{abstract}

Keywords - Identity Card Detection, Object Detection, YOLOv3 Model, Corner Detection, Image Rectification

\section{INTRODUCTION}

Based on the development of technology in Indonesia, object detection became one of the indispensable technological needs. Object detection is a very important development field in Indonesia, because the detection technology can be applied for identification verification based on object detection through Identity card data that is collected from Indonesian citizens. From the data collected by Badan Pusat Statistik (Central Institution of Statistic) in Indonesia, it is stated that Indonesia has a very high number of population and it is increasing every year.

Indonesia also has high growth rate numbers [1]. It shows that the population growth rate has reached 2.803.120 individuals per year. It means more citizen's data needs to be verified. It also means that the need of Identity card detection systems is absolute and important for the aspect of efficiency and accuracy for collecting and verifying identification data.
In recent years, the technological development in field object detection has had great success. Many algorithms have been published and used in various implementation fields whether it's a single-stage algorithm or two-stage algorithm. This studies in the field of object detection are aiming to get optimal results in terms of speed and accuracy.

There have been a lot of tests by many studies that show the existence of a single-stage algorithm for object detection such as YOLO (You Only look Once) and SSD (Single Shot Detector) may surpass the commonly used two-stage algorithm such as CNN (Convolutional Neural Networks), RCNN (Region-based Convolutional Neural Networks), and many others in terms of speed and accuracy. Inspired by many studies, we decided to use the enhancement of the YOLO algorithm called the YOLOv3.

In order to get the correct detection of the Identity card, we also use corner detection and image rectification on our image. From this, we get to detect the Identity card to its real shape even if the card was being held by hand and was slightly tilted in the photograph

\section{RELATED WORKS}

An example of application in object detection was a document detection by contours and contrast in MIDV- 500 dataset and SmartDoc dataset was done [2]. After some data pre-processing, they first do an edge extraction that results in two edge maps that will generate "mostly horizontal" and "mostly vertical" edges respectively. Each edge map is then blurred with a Gaussian filter. The vertical edge maps are then divided into three equal parts by horizontal cuts to improve short sides detection quality. Then with Fast Hough Transform (FHT), search line candidates via edge maps were applied. This results in 15 extreme points for horizontal FHT image and 45 for vertical FHT image, which is then used for brute-force to search two pairs of lines (vertical and horizontal). From this, they were able to decrease the total error by $9.73 \%$ from the original contour. 


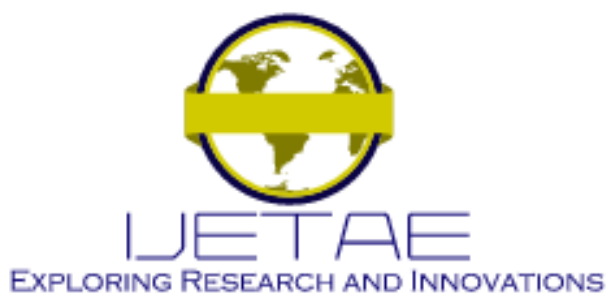

International Journal of Emerging Technology and Advanced Engineering Website: www.ijetae.com (E-ISSN 2250-2459, Scopus Indexed, ISO 9001:2008 Certified Journal, Volume 12, Issue 02, February 2022)

There are two types of object detection algorithm, One Stage Algorithm and Two Stage Algorithm [3]. From the research, Two Stage Algorithms are accuracy oriented and it affects the detection speed. The detection speed from Two Stage Algorithms is relatively slow, while One Stage Algorithms are relatively fast in terms of detection speed. But the weakness of the One Stage is the accuracy relatively below the Two Stage. The good news is there is improvement from the One Stage algorithm called YOLOv3. YOLOv3 has fast detection speed just like the characteristic of One Stage algorithm. But the accuracy of YOLOv3 can offset the Two Stage algorithm like Faster RCNN.

YOLOv3 has the excellence for its performance in terms of Speed Detection and Accuracy in rapid detection case [4] compared to SSD and Faster R-CNN. This is the reason why we choose YOLOv3 as our proposed method. YOLOv3 models detect small components like electronic components in [4] and [5]. It means, YOLOv3 is also capable of detecting small feature details in an object. YOLOv3 is built with special network architecture inspired by the feature pyramid network. It also has enhancement from the older version to handle tiny object detection problems and optimize the accuracy [6]. YOLOv3 performance is also mentioned in [7], [8], and [9].

Chadalawada S. K., did an object detection to detect vehicles with 3 different algorithms [10] to see which algorithm got the highest F1 score and fitted for the project that he was doing. The author uses 1097 image dataset containing images of Hauler, Excavator, Wheeled Loader, and the three vehicles combined. This dataset then got augmented to increase the number of dataset images. The total image dataset that was collected was 5485 images. These images are then trained using Faster-RCNN, YOLOv3, and tiny-YOLO. The author then calculated the F1 score of each algorithm and got 0.9 for the FasterRCNN algorithm, 0.9362 for YOLOv3 algorithm, and 0.8278 for the tiny- YOLO algorithm. From these results, the author suggested the use of YOLOv3 to be the algorithm used to detect the vehicle

One of the core processes of this project is detecting edges. By detecting edges, the corner and contour approximation will be more optimal. In the edge detection process, reducing the amount of data will be applied so the analysis of the image will be more simplified [11]. At the same time, edge detection produces structural information about object boundaries.
Canny method focuses on the specification of detection and localization criteria in a mathematical form

There are several edge detection algorithms such as Sobel, Robert Cross, Prewitt, and Canny [12]. These 4 are compared and showed that Canny has the advantage of using probability to find error rate, localization and response, better at detecting edges, and robust to overcome noises in an image.

YOLOv3 and Canny can become a great combination in terms of detecting objects. These two methods are combined and make a great performance result in detecting objects [13]. The fast detection speed and the accurate edge detection is the reason why this method is powerful

Scanning document technology has been widely used for different interests [14]. This technology is used for document transmission, pre-processing documents, and improving the quality of document images. By scanning documents, the rectification process is one of the core processes in the task. Document images may suffer perspective distortion when captured [15]. This problem becomes the challenge for the rectification process. Rectification uses 4 corner points to determine the optimal location of the object that wants to be rectified

\section{METHOD}

In detecting an ID card or identity card, we do a few processes in order to achieve the desired goal, which is to detect the ID card exactly to the shape. First, we do object detection with YOLOv3 to find the location of the ID card. Then, we do corner detection to get the real corner of the ID card. Finally, we do rectification on the detected ID card to turn the ID card into a readable picture.

\section{A. Object Detection}

In this project, we choose YOLOv3 as a method for object detection. YOLOv3 is an upgraded version of YOLO (You Only Look Once) [16], a real time object detector made by Joseph Redmond and Alif Farhadi. YOLO is well known for its efficiency and capability to detect objects in a short amount of time. YOLOv3 itself has many configurations such as YOLOv3-320, YOLOv3-416, YOLOv3-608, YOLOv3-tiny, and YOLOv3-spp.

Since the image size of our dataset is not consistent, in this project we decided to use YOLOv3-416 as the configuration to do our object detection. In this configuration, the image size that was used for the training and testing is $416 \times 416$. 


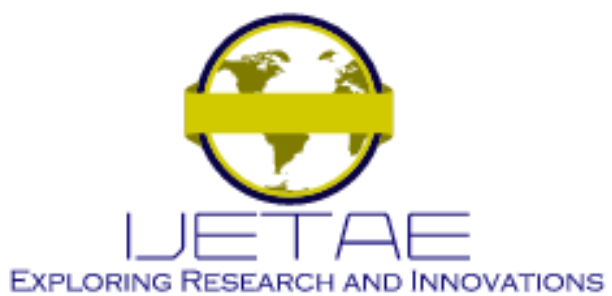

International Journal of Emerging Technology and Advanced Engineering Website: www.ijetae.com (E-ISSN 2250-2459, Scopus Indexed, ISO 9001:2008 Certified Journal, Volume 12, Issue 02, February 2022)

If the image is bigger than the $416 \times 416$, the image will be resized into smaller size without removing the image original ratio and then the empty space will be filled with white space to get the $416 \times 416$ size. If the image is smaller than the $416 \times 416$, the image will be resized into larger size without removing the image original ratio and then the empty space will also be filled with the white space. With this method, all of the image sizes will be the same but the quality of each image also changes.

Each of these resized images had to be labelled previously to set the ground truth of the Identity Card. This label was defined as a .txt file for each image which filled with bounding box data written in a format of (object class, object coordinates $\mathrm{x}$, object coordinates $\mathrm{y}$, width, height).

The whole process of YOLOv3 will accept input in a batch of image of shape (m, height, width, 3) and gives output of bounding box each in a shape of $(\mathrm{m}$, bounding box $\mathrm{x}$-coordinates, bounding box $\mathrm{y}$-coordinates, bounding box height, bounding box width, c).

The resized images will then enter the network of YOLOv3 with a three times up sample. Each of these up sample increases the feature map by twice the original size. The reason behind these three times up samples is because YOLOv3 does the object detection in three different scales.

In a $416 \times 416$ image size that we use and with 32 strides, the resultant feature map would be the size of $13 \mathrm{x}$ 13. Therefore, the first detection in YOLOv3 happened in a $13 \times 13$ feature map, then up sampled to $26 \times 26$ feature map, and another up sampled to a 52 x 52 feature map.

Each of these sizes of feature map affect the capability of YOLOv3 in detecting different sizes of objects. The $13 \mathrm{x}$ 13 feature map responsible for the object detection of large size objects. The $26 \times 26$ feature map responsible for the object detection of medium size objects. While the $52 \times 52$ feature map responsible for the object detection of small size objects.

For the detection kernel, YOLOv3 use 1 x 1 x (B x $(5+$ C)) as the shape, with "B" as the number of bounding boxes a cell can predict, " 5 " as the 4 attribute of bounding box and 1 object confidence, and " $\mathrm{C}$ " as the total number of classes.

With these 3 different scales detection in YOLOv3, the total bounding boxes that are predicted by YOLOv3 increase by ten times of the total bounding boxes that can be predicted by YOLOv2.
Inside these feature maps, the center of the ground truth box (cell) is chosen to be the one that is responsible for predicting the object. Inside each cell in the feature map there are anchor boxes with the same values in which got flattened by multiplying the total anchor boxes with their values. These cells then get computed to find the probability that the cells contain a certain class or an object.

$$
\begin{gathered}
b_{x}=\sigma\left(t_{x}\right)+c_{x} \\
b_{y}=\sigma\left(t_{y}\right)+c_{y} \\
b_{w}=p_{w} e^{t_{w}} \\
b_{h}=p_{h} e^{t_{h}}
\end{gathered}
$$

Where $b_{x}, b_{y}, b_{w}, b_{h}$ are the $x$-coordinates center, $y$ coordinates center, width, and height of the prediction and the $t_{x}, t_{y}, t_{w}, t_{h}$ was for the network output. While the $c_{x}$ and $c_{y}$ are the top left coordinates of the grid and $p_{w}$ and $p_{h}$ was the anchor dimension for the box. The output then got through the sigmoid function in order to keep the center in the grid.

Since the 3 scales detection in YOLOv3 increase the number of predicted bounding box, we need to filter these bounding boxes by selecting bounding boxes with the highest confidence and reduce the amount of bounding boxes by choosing only one bounding boxes when there are bounding boxes that overlap each other and detect the same object by using NMS (Non Max Suppression).

NMS uses a function called IoU (Intersection over Union) where we need to find the intersection area and union area of each bounding box and divide them. The result from doing NMS can be seen in Fig. 1.

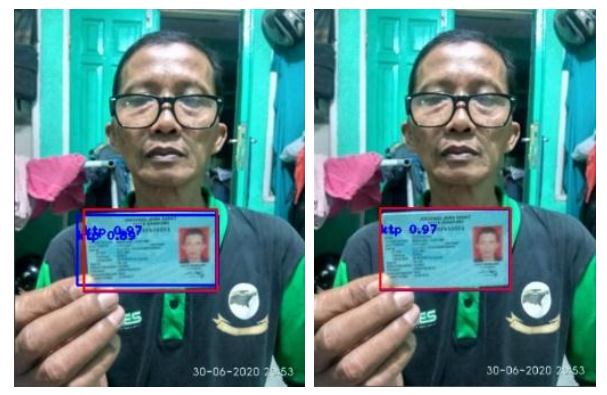

FIGURE 1. Detection Result using YOLOv3 Before NMS (left) and After NMS (right). 


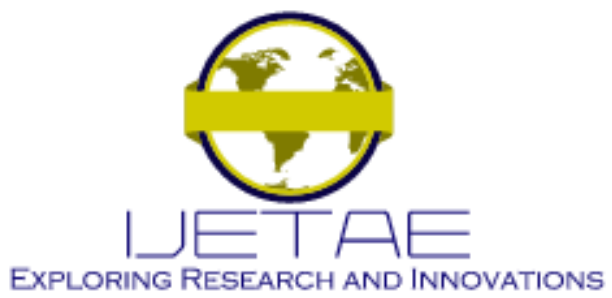

International Journal of Emerging Technology and Advanced Engineering Website: www.ijetae.com (E-ISSN 2250-2459, Scopus Indexed, ISO 9001:2008 Certified Journal, Volume 12, Issue 02, February 2022)

There are a variety of backbones that can be used, the difference of performance that has been stated in previous study [16] shows that the previous Darknet-19 that was made for YOLOv2, manage to get 171 FPS which is $222 \%$ faster than Resnet-101 and 362\% faster than Resnet- 152 . But the accuracy that can be achieved by Darknet-19 was below the accuracy that can be achieved by Resnet-101 and Resnet-152.

This was happened because of the architecture of YOLOv2. YOLOv2 itself is superfast network affected by the layers. There are only 19 convolutional layers and 5 max pooling layers in Darknet-19. In YOLOv2 loss function, the last three terms are the squared errors which will be replaced by cross-entropy error terms. The minor shortfall of YOLOv2 is it stills softmaxing the class scores. By softmaxing, it means if an object belongs to one class, it can't be in another. Besides, The complexity of Darknet-19 is relatively low.

Meanwhile Darknet-53 that was made for YOLOv3, was able to achieve the same performance as Resnet-152 with $1457 \mathrm{BFLOP} / \mathrm{s}$ (Billion Floating Point Operations per seconds) which is 17\% higher than Darknet-19 1246 BFLOP/s. This resulted in the capability of Darknet-53 to detect with 93.8 accuracy in 78 FPS. Based on these data performances, we decided to use Darknet-53 as the backbone that we use in YOLOv3 object detection. As the Darknet-53 has a great accuracy, high billion floating point operations per second, and a decent FPS for object detection. Darknet-53 is a convolution neural network updated from Darknet-19. The Darknet-53 is made out of 53 convolutional layers as described in Fig. 2.

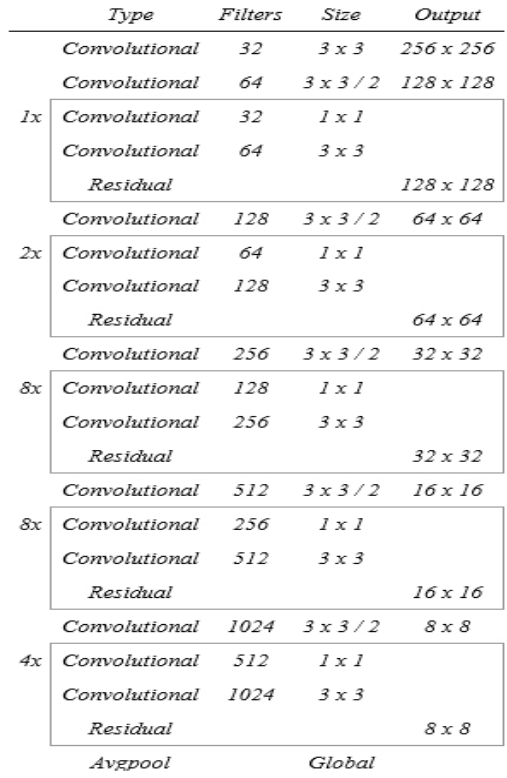

FIGURE 2. DARKNET-53[16].

For the detection task itself, YOLOv3 uses another additional 53 layers stacked onto the backbone making it have a 106 of layers in total. These layers are the reason for smaller FPS in YOLOv3 detection compared to FPS in YOLOv2 detection.

YOLOv3 models that detect the Identity Card from an image will be a great feature to localize the location of the card in the image. With this ability the corner detection will be more accurate caused by the minimization area to be calculated. The result from Identity Card Localization can be seen in Fig. 3 . 


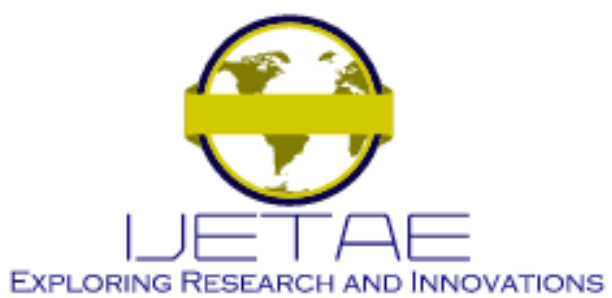

International Journal of Emerging Technology and Advanced Engineering Website: www.ijetae.com (E-ISSN 2250-2459, Scopus Indexed, ISO 9001:2008 Certified Journal, Volume 12, Issue 02, February 2022)
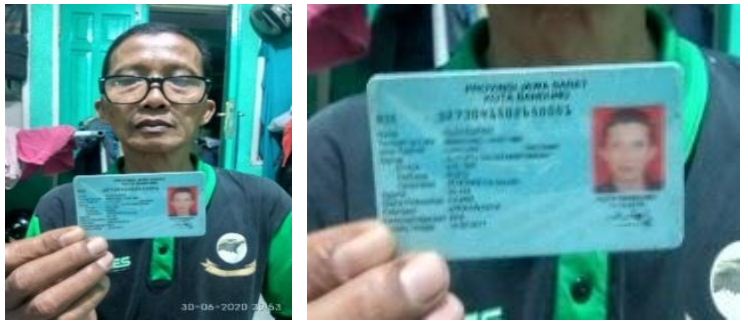

FIGURE 3. Identity Card Localization by YOLOv3.

\section{B. Corner Detection}

In this project, we detect the corners of the ID card by finding the contour of the ID card image. The images that are used in this process are the localized image. Contour is a curve joining all the continuous points (along the boundary), having the same color or intensity. Contour can be defined as the shape or the outline of something. But, before finding the contours, there are several preprocessing steps that should be done. To find the contours we need a binary image for better performance in detecting. The image will be filtered with Bilateral Filter.

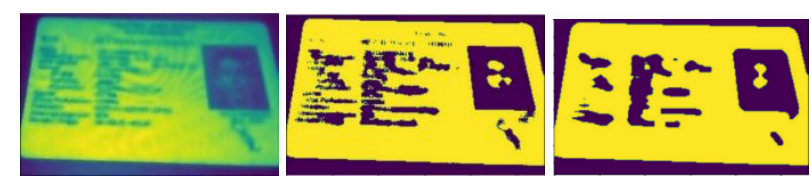

FIGURE 4. Bilateral Filtered Image(Left), Image with Adaptive Threshold(Middle), Median Filtered Image(Right)

The next process is the thresholding process. By thresholding, there will be a white and black image produced. Adaptive Thresholding was chosen because if a global value is used for the threshold value, sometimes the result is not good enough, affected by different lighting compositions in different areas in an image. The threshold value is the weighted sum of neighbourhood values where weights are a gaussian window. After the thresholding process finished, the images are filtered by Median Filter to clear the small details. Median filter computes the median of all the pixels under the kernel window and the central pixel is replaced with this median value. This process can be seen in Fig. 4.

Then, the process that should be done is detecting edges in the image. The Canny edge detection algorithm was chosen to detect edges. By using Canny, we have to specify three values called hysteresis thresholding, which separates edges in an image into three groups such as edges, not edges, and something in between. There are several processes in the Canny edge detection algorithm.
The first process is the noise removal by using $5 \times 5$ Gaussian Filter. Then, the smoothened image was filtered with Sobel kernel in horizontal and vertical direction. This process will produce two first derivatives in horizontal and vertical direction. From these two, we can find edge gradient and direction for each pixel. This calculation were performed using Equation 5 and 6.

$$
\begin{aligned}
& \text { Edge Gradient }(G)=\sqrt{G_{x}^{2}+G_{y}^{2}} \\
& \qquad \text { Angle }(\theta)=\tan ^{-1}\left(\frac{G_{y}}{G_{x}}\right)
\end{aligned}
$$

After the gradient magnitude and direction are collected, the image was scanned to remove the unwanted pixels that do not represent the edges. In this process, each pixel is checked whether it is local maximum in its neighbourhood or not. This process is called the non-maximum suppression. The last process in Canny edge detection is Hysteresis Thresholding. This process decides which are the true edges or not in the image. We will use two threshold values that are minVal and maxVal. These two threshold values will be the decision maker whether it is definitely an edge or not. All the processes above produce the strong edges in the image. A sample of output image after applying Canny Edge detection is shown in Fig. 5.

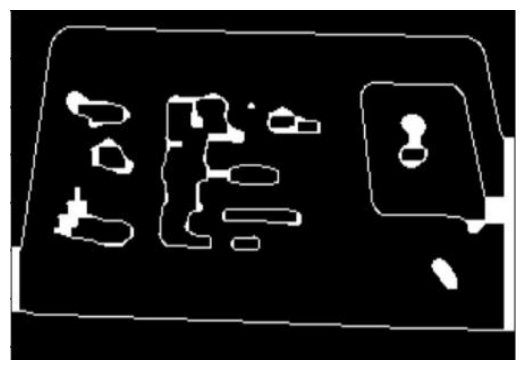

FIGURE 5. Edge Detection using Canny.

After the edge detection in an image already done, the processed image is used to find the contour approximation from the image. The processed image is an image divided into two kinds of pixels, black and white pixels. White pixels representing the edges and the black pixels representing the background. From the array of contours we get, the program will go through all the contours and every time the criteria is met, it will be saved. This process will be repeated until the biggest contour has been found 


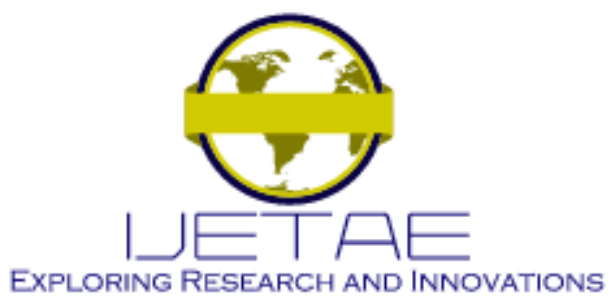

International Journal of Emerging Technology and Advanced Engineering Website: www.ijetae.com (E-ISSN 2250-2459, Scopus Indexed, ISO 9001:2008 Certified Journal, Volume 12, Issue 02, February 2022)

\section{Rectification}

The next step after the page contour and the 4 corners have been found is to rectify the image based on the corners. But, before this step the points have to be offset and rescaled to the original size. After the previous process is done, the four corners will be used as the parameter to do the perspective transformation. The points from the image will be defined as the source points and the points that will be applied from the transformation process will be declared as target points. For perspective transformation, a $3 \times 3$ transformation matrix will be needed. After the points were defined, the warping process must be applied for getting the result of the perspective transformation. A sample result of the rectification process is shown in Fig. 6 .
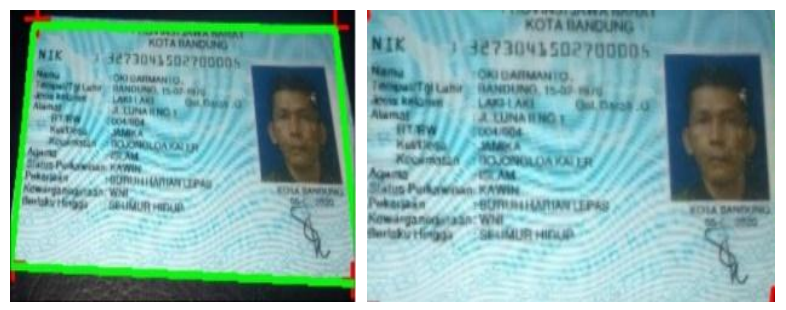

FIGURE 6. Result Example of Rectification Process.

\section{EXPERIMENTS}

In detecting an ID card or identity card, we do a few processes in order to achieve the desired goal, which is to detect the ID card exactly to the shape. First we do object detection with YOLOv3 to find the location of the ID card. To do this, we use Google Colab with GPU Nvidia K80 and 12GB RAM. Then, we do corner detection to get the real corner of the ID card. Finally, we do rectification on the detected ID card to turn the ID card into a readable picture. The process of corner detecting and rectification was done in Visual Studio Code with GPU GTX $1650 \mathrm{Ti}$ and 8GB RAM. All of the process was done in Python environment.

\section{A. Dataset}

In this project, we are given a credential dataset which contains a total of 183 images of ID cards varying from the clear and close picture of the ID card to blurred and far ID cards which are usually held by hand. The dataset then split into 100 train dataset, 28 validation dataset, and 57 test dataset.
Since these pictures are taken with various cameras therefore the quality of each picture is different. The size of each picture is also different since every picture was taken by a different photographer and with different devices. In the data processing, we change the file structure into an easier file structure for the program to run. We also did some labelling on each image to set the ground truth for object detection and corner detection.

\section{B. Experimental Design}

In this project, we decided to train our dataset with $416 \mathrm{x}$ 416 width $\mathrm{x}$ height, 0.9 momentum, 1 mini batch, 16 batch, and 0.5 threshold. We also did some hyperparameter tuning by using 2 types of learning rate which is 0.05 and 0.001 , and 2 types of optimizers, which is SGD+momentum+decay optimizer and adam optimizer. SGD is one of the variants from Gradient Descent. The main idea of SGD is removing the redundancy and inefficiency by computing small subset or random selection of data examples rather then the whole dataset. In this project, the error from the corner detection will be calculated as well. The calculation is based on the distance difference between the corners that have been detected and the corners from the ground truth on the image. The ground truth is processed through manual labelling from the image based on the corners' coordinates. The distance calculation method that was used in this project is the Euclidean distance.

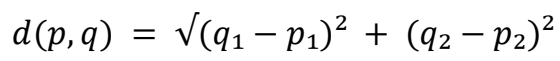

The formula in Equation 7 will be used through an image and calculate all the corner points distance to all coordinates in ground truth corner points to see the average distance error in an image. The process will be applied to the 57 test images to see the average performance.

\section{Experimental Results}

The validation was performed to tune the hyperparameters of the YOLOv3 using the validation dataset. The validation results of the object detection is summarized in Table I. With 1500 iterations, the YOLOv3 object detection with adam optimizer got $4 \%$ of mAP in 0.05 learning rate and $83.3 \%$ of $\mathrm{mAP}$ in 0.001 learning rate. Meanwhile using the SGD optimizer made the YOLOv3 object detection got $86 \% \mathrm{mAP}$ in 0.05 learning rate and $91.1 \% \mathrm{mAP}$ in 0.001 learning rate. 


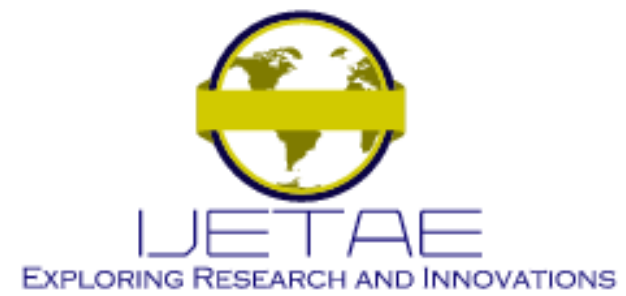

International Journal of Emerging Technology and Advanced Engineering Website: www.ijetae.com (E-ISSN 2250-2459, Scopus Indexed, ISO 9001:2008 Certified Journal, Volume 12, Issue 02, February 2022)

Table I

Detection Validation Performance

\begin{tabular}{|c|c|c|}
\hline Optimizer & Adam & SGD \\
\hline $\mathbf{0 . 0 5}$ learning rate & $4 \%$ & $86 \%$ \\
\hline $\mathbf{0 . 0 0 1}$ learning rate & $83.3 \%$ & $\mathbf{9 1 . 1 \%}$ \\
\hline
\end{tabular}

With these validation performances, we decided to use the SGD optimizer on 0.001 learning rate since this method got the highest mAP. From this configuration, we get to achieve $92.59 \%$ mAP in the test dataset with 5 second total detection time.In the project, the error calculation in terms of distance between the corner that is detected and the corner from ground truth have to be measured as well. The dataset that was used for the test is 57 images that have been localized by YOLOv3 algorithm. As shown in Table II, the average error of the corner detection is 37.06 pixels.

Table II

Corner Detection Performance

\begin{tabular}{|l|l|}
\hline Images & Averages Error \\
\hline Adam & 37.06 \\
\hline
\end{tabular}

\section{CONCLUSIONS AND FUTURE WORK}

From the experiment that has been done, we are able to see that YOLOv3 is a good object detection algorithm that can be used to detect Identity cards from images. We can also conclude that the smaller learning rate that was used, will increase object detection accuracy, and decrease total detection time. The corner detection result that is used for image rectification shows this method has a pretty good result. The average of distance error reaches only 37 pixels from 4 corners in an image. This result is affected by the quality of the image and the ID card location in the image. In the future, this identity card detection system may need an upgrade in the object detection process to get higher accuracy and faster time detection. This can be achieved by changing the YOLOv3 algorithm or upgrading the Darknet53 backbone. The average distance error in the edge detection may also get reduced with other edge detection algorithms. We believe more structured dataset will also increase the accuracy of the whole identity card detection process.

\section{REFERENCES}

[1] Badan Pusat Statistik. (2021). Jumlah Penduduk Hasil Proyeksi Menurut Provinsi dan Jenis Kelamin (Ribu Jiwa), 2015-2017. https://www.bps.go.id/indicator/12/1886/2/jumlah-penduduk-hasilproyeksi-menurut-provinsi-dan-jenis-kelamin.html
[2] Tropin, Daniil V., Ilyuhin, Sergey A., Nikolaev, Dmitry P., Arlazarov, Vladimir V. 2020. Approach for Document Detection by Contours and Contrasts.

[3] Wang, H., Yu, Y. I., Cai, Y., Chen, X., Chen, L., \& Liu, Q. (2019b). A Comparative Study of State-of-the-Art Deep Learning Algorithms for Vehicle Detection. IEEE Intelligent Transportation Systems Magazine, 11(2), 82-95.doi: 10.1109/mits.2019.2903518.

[4] Huang, R., Gu, J., Sun, X., Hou, Y., \&amp; Uddin, S. (2019). A Rapid Recognition Method for Electronic Components Based on the Improved YOLO-V3 Network.Electronics, 8(8).825. doi:10.3390/electronics8080825.

[5] Li, J., Gu, J., Huang, Z., \& Wen, J. (2019). Application Research of Improved YOLO V3 Algorithm in PCB Electronic Component Detection. Applied Sciences, 9(18), 3750. https://doi.org/10.3390/app9183750.

[6] Rajendran, S. P., Shine, L., Pradeep, R., \& Vijayaraghavan, S. (2019). Real-Time Traffic Sign Recognition using YOLOv3 based Detector. 2019 10th International Conference on Computing, Communication and Networking Technologies (ICCCNT). https://doi.org/10.1109/iccent45670.2019.8944890.

[7] Wu, F., Jin, G., Gao, M., He, Z., \&amp; Yang, Y., (2019). Helmet Detection Based On Improved YOLO V3 Deep Model.

[8] Wu, S., \& Zhang, L. (2018). Using Popular Object Detection Methods for Real Time Forest Fire Detection. 2018 11th International Symposium on Computational Intelligence and Design (ISCID), 280- 284. https://doi.org/10.1109/iscid.2018.00070.

[9] Fachrie, M. (2020). A Simple Vehicle Counting System Using Deep Learning with YOLOv3 Model. Jurnal RESTI (Rekayasa Sistem Dan Teknologi Informasi), 4(3), 462-468. https://doi.org/10.29207/resti.v4i3.1871

[10] Canny, J. (1986). A Computational Approach to Edge Detection. IEEE Transactions on Pattern Analysis and Machine Intelligence, PAMI-8(6),

[11] Chadalawada, S. K. (2020). Real Time Object Detection and Recognition Using Deep Learning Methods

[12] Igbinosa, I. E. (2015). Comparison of Edge Detection Technique in Image Processing Techniques. Information Technology \& Electrical Engineering, 2(1), 25-29..

[13] Chung, Y., \&amp; Lin, C. (2020). Application of a model that combines the yolov3 object detection algorithm and canny edge detection algorithm to detect highway accidents. Symmetry, 12(11), 1875. doi:10.3390/sym 12111875

[14] Lu, S., Chen, B. M., \& Ko, C. C. (2005). Perspective rectification of document images using fuzzy set and morphological operations. Image and VisionComputing, 23(5), 541-553. https://doi.org/10.1016/j.imavis.2005.01.003.

[15] Miao, L., \& Peng, S. (2006). Perspective Rectification of Document Images Based on Morphology. 2006 International Conference on Computational Intelligence and Security, 1805-1808. https://doi.org/10.1109/iccias.2006.295374.

[16] Redmon, Joseph and Farhadi, Ali. 2018. YOLOv3: An Incremental Improvement. 